\title{
Stability Analysis and Control Optimization of a Prey-Predator Model with Linear Feedback Control
}

\author{
Yaning Li, ${ }^{1}$ Yan Li $\left(\mathbb{D},{ }^{2}\right.$ Yu Liu, ${ }^{3}$ and Huidong Cheng $\mathbb{D}^{1}$ \\ ${ }^{1}$ College of Mathematics and Systems Science, Shandong University of Science and Technology, Qingdao 266590, China \\ ${ }^{2}$ College of Electrical Engineering and Automation, Shandong University of Science and Technology, Qingdao 266590, China \\ ${ }^{3}$ College of Foreign Languages, Shandong University of Science and Technology, Qingdao 266590, China \\ Correspondence should be addressed to Huidong Cheng; chd900517@sdust.edu.cn
}

Received 13 July 2018; Revised 8 November 2018; Accepted 26 November 2018; Published 5 December 2018

Guest Editor: Abdul Qadeer Khan

Copyright (c) 2018 Yaning Li et al. This is an open access article distributed under the Creative Commons Attribution License, which permits unrestricted use, distribution, and reproduction in any medium, provided the original work is properly cited.

\begin{abstract}
The application of pest management involves two thresholds when the chemical control and biological control are adopted, respectively. Our purpose is to provide an appropriate balance between the chemical control and biological control. Therefore, a Smith predator-prey system for integrated pest management is established in this paper. In this model, the intensity of implementation of biological control and chemical control depends linearly on the selected control level (threshold). Firstly, the existence and uniqueness of the order-one periodic solution (i.e., OOPS) are proved by means of the subsequent function method to confirm the feasibility of the biological and chemical control strategy of pest management. Secondly, the stability of system is proved by the limit method of the successor points' sequences and the analogue of the Poincaré criterion. Moreover, an optimization strategy is formulated to reduce the total cost and obtain the best level of pest control. Finally, the numerical simulation of a specific model is performed.
\end{abstract}

\section{Introduction}

In the practical production, effective control of pests is a very important issue of the world, which catches attention of scholars for pest management method [1-7]. Integrated pest management (IPM), also known as integrated pest control (IPC), is an effective approach that integrates biological, chemical tactics, and physical methods for pests control [811]. Due to population dynamics and its related environment, IPM utilize effective methods and techniques comprehensively to reduce the level of economic harm caused by pests. The aim of IPM is to control the density of the insects under the economic threshold by integrated usage of less harmful pesticides and biological control methods for maximizing the protection of the ecosystem.

In mathematics, impulsive differential equations (IDES) is such a powerful tool to describe these phenomena that rapid changes in biological populations are caused by the variety of the pests control by artificial intervention [12-22]. In recent years, the theoretical studies on IDES have produced a lot of good research results [23-34]. Based on the theoretical research, some scholars have introduced impulsive differential equations in Lotka-Volterra system such as the regular release of predators [35-37]; the periodic release of infected pests [38-40]; the periodic release of predators together with regular spray of pesticides [41-43]; the periodic release of predators and infected pests together with regular spray of pesticides $[39,44]$. In the practical application, the two control measures can be adopted at two different levels of pest density concerning this case. Nie et al. [45], Tian et al. [46], Zhao et al. [47], and Zhang et al. [48] studied the following predator-prey system and assumed that different control measures were adopted at different thresholds,

$$
\begin{aligned}
u^{\prime}(t) & =a u \ln \left(\frac{K}{u}\right)-b u v, \\
v^{\prime}(t) & =v(t)(-d+\lambda b u), \\
\Delta u(t) & =0, \\
\Delta v(t) & =\gamma, h_{1}, h_{2}, \text { or } u=h_{1}, \quad v>v^{*},
\end{aligned}
$$




$$
\begin{aligned}
& \Delta u(t)=-\delta u(t), \\
& \Delta v(t)=-\varrho v(t)+\tau,
\end{aligned}
$$$$
u=h_{2} \text {, }
$$

where the intrinsic growth rate of prey is denoted by $a$, the environment carrying capacity is denoted by $K$, the predation rate by natural enemies is denoted by $b$, and the transformation rate and the death rate of predator are denoted by $\lambda$ and $d$, respectively. The $\eta$ is a positive parameter, and the effect of pesticide to predator and prey species is denoted by $\varrho$ and $\delta$, respectively. The releasing quantity of natural enemy $v(t)$ are denoted by $\gamma$ and $\tau$, respectively.

It is of great practical significance to adopt biological and chemical control strategies based on the different pest thresholds. But an important issue in this process should be pointed out, in which the biological control is carried out when the density of pest denoted by $u(t)$ reaches the threshold $h_{1}$, and when the density $u(t)$ reaches the threshold $h_{2}$, the integrated control strategy is adopted. But no strategy adopted for the density of pest denoted by $u(t)=h$, where $h_{1}<h<h_{2}$, which is obviously unreasonable. In addition, from an economic and practical point of view, the control taken at threshold $h_{1}$ seems to be early and the amount of releasing predators will also be huge, while the control taken at threshold $h_{2}$ seems to be late and the intensity of chemical control will also be high. Considering the above problems, we should choose a pest control method between $h_{1}$ and $h_{2}$.

An outline of this paper is as follows. In next section, a pest management Smith model is formulated. Then the existence, uniqueness, and the asymptotically orbit stability of order-one periodic solution (OOPS) of system (7) are proved in Section 3. In Section 4, an optimization problem is formulated and obtained the minimized total cost in pest control. The theoretical results are verified by numerical simulations in Section 5. Finally, a conclusion is drawn.

\section{Model Formulation}

In biological mathematics, Logistic model [10]

$$
\frac{d x}{d t}=r x\left(1-\frac{x}{K}\right)
$$

is a classical mathematical model, where the predator and prey densities at time $t$ are denoted by $y(t)$ and $x(t)$. $r$ denotes the intrinsic rate of growth and $K$ denotes the maximum environment carrying capacity, while system (2) is based on the assumption that the relative growth rate $d x / x d t$ of the population size is linear function $1-x / K$. In 1963, F.E.Smith found that the data about the population of Daphnia did not conform to the linear function [49]. Thus, Smith assumed that the relative growth rate of population density at time $t$ is proportional to the amount of remaining food; i.e.,

$$
\frac{1}{x} \frac{d x}{d t}=r\left(1-\frac{H(t)}{T}\right),
$$

where $H(t)$ is the rate of food demand of the population at time $t ; T$ is the rate of demand for food in a population saturated state. Smith assumed that the food required to keep the population is $c_{1} x(t)$ and the food required for the population to reproduce is $c_{2}(d x / d t)$. That is to say,

$$
H(x)=c_{1} x(t)+c_{2} \frac{d x}{d t} .
$$

Then

$$
\frac{d x}{d t}=r x\left(1-\frac{T-c_{1} x}{T+r c_{2} x}\right)
$$

Considering the demand for food of population reproduction, the Smith model uses the hyperbolic function ( $T$ $\left.c_{1} x\right) /\left(T+r c_{2} x\right)$ instead of the linear function in the Logistic model. Thus, the Smith model is a further improvement of Logistic model. With the absence of predators, the per capita growth rate $l_{\text {grow }}$ of the pest is assumed to be the Smith growth [49] model.

$$
l_{\text {grow }}=r x \frac{K-x}{K-(r / c) x} .
$$

By the control strategy, the following predator-prey Smith system is investigated in this paper:

$$
\begin{aligned}
& \frac{d x}{d t}=\frac{m x(t)-r x(t)^{2}}{K+d x(t)}-q x(t) y(t), \\
& \frac{d y}{d t}=\mu x(t) y(t)-l y(t),
\end{aligned}
$$$$
x<h,
$$

$$
\begin{aligned}
& \Delta x(t)=-\alpha(x) x(t), \\
& \Delta y(t)=-\beta(x) y(t)+\sigma(x),
\end{aligned}
$$

$$
x=h, y \leq \bar{y}_{h}
$$

where the releasing amount of the predator is denoted by $\sigma(x)$ and $\sigma\left(h_{1}\right)=\sigma_{\text {max }}, \sigma\left(h_{2}\right)=\sigma_{\text {min }}$, where $0 \leq \sigma_{\text {min }}<\sigma_{\text {max }}$. The strength of chemical control to the prey is $\alpha(x)$ and that to the predator is $\beta(x)$, where the parameters $\sigma(x), \alpha(x), \beta(x)$ are continuous functions and satisfies $\alpha\left(h_{2}\right)=\alpha_{\text {max }}, \beta\left(h_{2}\right)=$ $\beta_{\text {max }}$. A pest control level $h$ is between $h_{1}$ and $h_{2} \cdot \bar{y}_{h}$ denotes the level of the predator at a lower density. By calculation we obtain $\bar{y}_{h}=(\lambda K q-l) / q(K q \lambda-d l)$, where $r K \triangleq m, r / c \triangleq$ $d, \lambda q \triangleq \mu<l$ are constants. When the density of predator is below $\bar{y}_{h}$, the chemical control is taken. Clearly, the control strategy of system (7) changes into the biological control strategy of system (1) when parameters $\alpha(x), \beta(x)$, and $\sigma(x)$, $x$ of system (7), are chosen $0,0, \gamma, h_{1}$, respectively. When parameters $\alpha(x), \beta(x)$, and $\sigma(x), x$ of system (7), are chosen $\delta$, $\varrho, \tau h_{2}$, respectively, the control strategy of system (7) turns into the integrated control strategy of system (1). Therefore, system (7) is the further promotion of system (1). 
In our paper, $\sigma(x), \alpha(x)$, and $\beta(x)$ are assumed to have the following linear form [10]

$$
\begin{aligned}
& \sigma(x)=\sigma_{\text {max }}-\left(\sigma_{\text {max }}-\sigma_{\text {min }}\right) \frac{x-h_{1}}{h_{2}-h_{1}}, \\
& \alpha(x)=\alpha_{\text {max }} \frac{x-h_{1}}{h_{2}-h_{1}} \\
& \beta(x)=\beta_{\text {max }} \frac{x-h_{1}}{h_{2}-h_{1}}
\end{aligned}
$$

\section{Dynamical Analysis of System (7)}

In this section, we dynamically analyze system (7) to study the existence, uniqueness and orbital asymptotical stability of the OOPS. For convenience, OOPS is used to represent the orderone periodic solution.

3.1. Qualitative Analysis of System (7). We first study the following continuous system of system (7); i.e.,

$$
\begin{aligned}
& \frac{d x(t)}{d t}=\frac{m x(t)-r x(t)^{2}}{K+d x(t)}-q x(t) y(t), \\
& \frac{d y(t)}{d t}=\mu x(t) y(t)-l y(t) .
\end{aligned}
$$

Let

$$
\begin{array}{r}
\frac{m x(t)-r x(t)^{2}}{K+d x(t)}-q x(t) y(t)=0, \\
\mu x(t) y(t)-l y(t)=0 .
\end{array}
$$

Then we get three equilibria $O(0,0), E_{0}(m / r, 0)$, and $E^{*}\left(x^{*}, y^{*}\right)$, where

$$
\begin{aligned}
& x^{*}=\frac{l}{\mu}, \\
& y^{*}=\frac{\mu m-l r}{q(\mu K+d l)} .
\end{aligned}
$$

Let

$$
\text { (I) }: \frac{l}{\mu}>\frac{\sqrt{\Delta}-r K}{r d},
$$

where $\Delta=r^{2} K^{2}+m K r d$. Thus, we get the following theorem.

Theorem 1. The positive equilibrium point $E^{*}\left(x^{*}, y^{*}\right)$ is locally asymptotically stable, if (I) holds.

Proof. At the point $E^{*}\left(x^{*}, y^{*}\right)$, the Jacobian matrix is

$$
J\left(E^{*}\right)=\left(\begin{array}{cc}
\frac{m K-r x^{*}\left(d x^{*}+2 K\right)}{\left(K+d x^{*}\right)^{2}}-q y^{*} & -q x^{*} \\
\mu y^{*} & 0
\end{array}\right),
$$

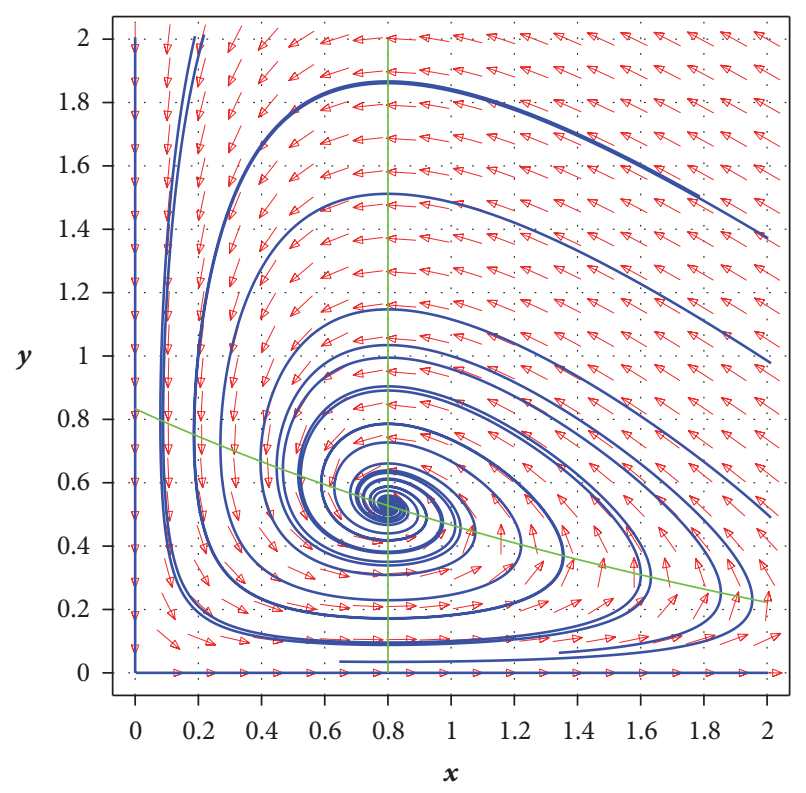

FIGURE 1: The phase diagram of system (7) with $m=1, r=0.3$, $K=2, d=0.5, q=0.6, \mu=0.5$, and $l=0.4$.

then

$$
\begin{aligned}
\operatorname{det}\left(J\left(E^{*}\right)\right) & =q \mu x^{*} y^{*}>0, \\
\operatorname{tr}\left(J\left(E^{*}\right)\right) & =\frac{m K-r x^{*}\left(d x^{*}+2 K\right)}{\left(K+d x^{*}\right)^{2}}-q y^{*} .
\end{aligned}
$$

When (I) holds, then $\operatorname{tr}\left(J\left(E^{*}\right)\right)<0$. Thus the point $E^{*}$ is locally asymptotically stable.

Theorem 2. If $\mu x \leq l$ holds, then the point $E^{*}$ is globally asymptotically stable.

Proof. Let $B=1 / x$, then we have

$$
\begin{aligned}
D & =\frac{\partial(P B)}{\partial x}+\frac{\partial(Q B)}{\partial x} \\
& =\frac{-r(K+d x)-d(m-r x)}{(K+d x)^{2}}+\mu-\frac{l}{x} \\
& =\frac{-r K-d m}{(K+d x)^{2}}+\mu-\frac{l}{x}
\end{aligned}
$$

when $\mu x \leq l$, then $D<0$.

By the method in [48], the point $E^{*}\left(x^{*}, y^{*}\right)$ is globally asymptotically stable (see Figure 1.)

3.2. Existence and Uniqueness of the Periodic Orbit of System (7). For convenience, let $H(x, y)=H_{0}$ denote the first integral of system (7), where the implicit function $H(x, y)=$ $H\left(x_{0}, y_{0}\right)$ is divided into upper and lower branches by isoclinic line $d x / d t=0$ denoted by $y_{H^{+}}\left(x, P_{0}\right)$ and $y_{H^{-}}\left(x, P_{0}\right)$, where the starting point is $P_{0}$. The impulsive set of system (7) is denoted by $\sum_{M}=\left\{(x, y) \mid x=h, 0 \leq y \leq \overline{y_{h}}\right\}$ and the phase set is denoted by $\sum_{N}=\{(x, y) \mid x=(1-\alpha(h)) h, y \geq 0\}$. 


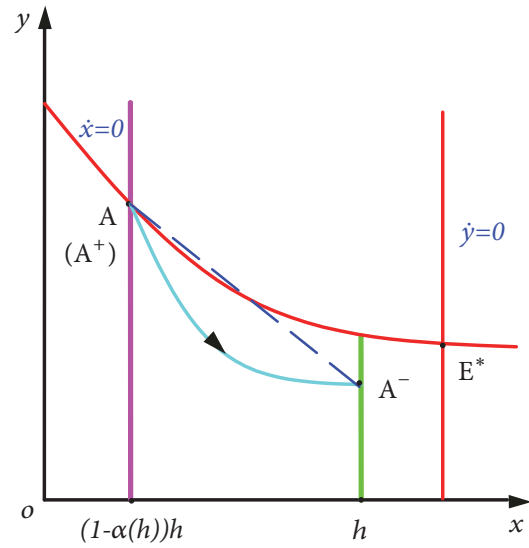

(a)

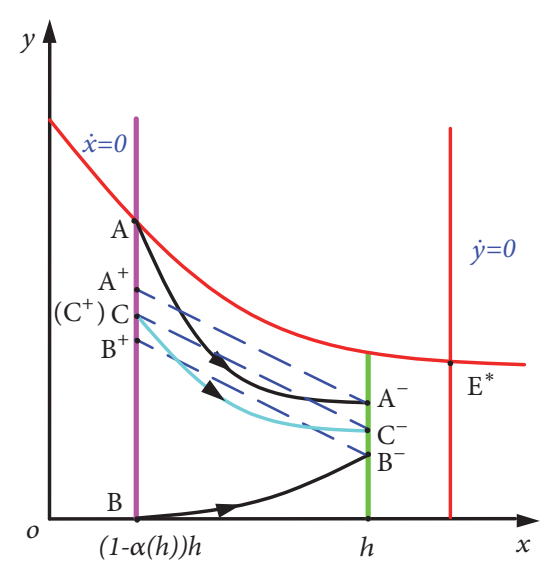

(b)

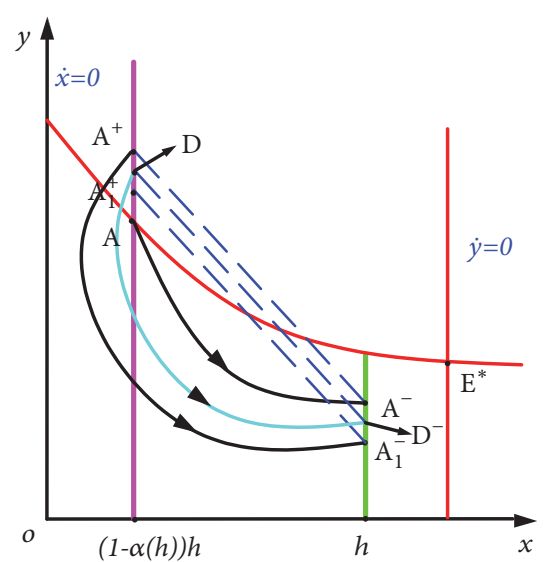

(c)

Figure 2: The existence of the OOPS of system (7). (a) Discussion in Case (i) if $\sigma(h)=\bar{\sigma}$. (b) Discussion in Case (i) if $\sigma(h)<\bar{\sigma}$. (c) Discussion in Case (ii).

Let $L_{1}=\{(x, y) \mid x=l / \mu, y \geq 0\}$ and $L_{2}=\{(x, y) \mid 0 \leq$ $x, y=(m-r x) / q(K+d x)\}$ as the isoclinic line $d y / d t=0$ and $d x / d t=0$, respectively. For any point $A\left(x_{A}, y_{A}\right)$, where $x_{A}$ and $y_{A}$ are denoted as the abscissa and ordinate of point $A$, respectively. By the location of the threshold $h$ and positive equilibrium point $E^{*}$, we get the following theorem.

Theorem 3. If $0<h_{1}<h<\min \left\{x^{*}, h_{2}\right\}$ holds, then $a$ uniqueness OOPS exists in system (7).

\section{Proof.}

Case I $\left(0<h_{1}<h<\min \left\{x^{*}, h_{2}\right\}\right)$. In view of Theorems 1 and 2 , for any point $Q_{0} \in \Omega$ where $\Omega=\{(x, y) \mid 0 \leq$ $x \leq(1-\alpha(h)) h, y \in R\}$ the trajectory $y_{H^{-}}\left(x, Q_{0}\right)$ has an intersection point with phase set $\sum_{N}$. Thus, we discuss the trajectory tendency of the initial point on the phase set $\Sigma_{N}$.

Assuming the intersection point of phase set $\sum_{N}$ and isoclinic line $d x / d t=0$ is point $A\left(x_{A}, y_{A}\right)$, where $x_{A}=$ $(1-\alpha(h)) h$ and $y_{A}=(m-r h(1-\alpha(h))) / q[K+d h(1-\alpha)]$. The trajectory $y_{H^{-}}((1-\alpha) h, A)$ intersects with pulse set $\Sigma_{M}$ at point $A^{-}\left(x_{A^{-}}, y_{A^{-}}\right)$, then the impulsive function can transfer the point $A^{-}\left(x_{A^{-}}, y_{A^{-}}\right)$into the point $A^{+}\left(x_{A^{+}}, y_{A^{+}}\right)$. Thus, we have

$$
\begin{aligned}
y_{A^{+}} & =(1-\beta(h)) y_{A^{-}}+\sigma(h) \\
& =(1-\beta(h)) y_{H^{-}}(h, A)+\sigma(h) ;
\end{aligned}
$$

define

$$
\bar{\sigma}(h) \triangleq y_{A^{+}}-(1-\beta(h)) y_{H^{-}}(h, A) .
$$

By the magnitudes between $\sigma(h)$ and $\bar{\sigma}(h)$, one has

(i) $\sigma(h) \leq \bar{\sigma}$.

If $\sigma(h)=\bar{\sigma}$, the subsequent function of point $A\left(x_{A}, y_{A}\right)$ is $g(A)=0$, then the trajectory $\widehat{A A^{-} A^{+}}$is an OOPS.

If $\sigma(h)<\bar{\sigma}$, the point $A^{+}\left(x_{A^{+}}, y_{A^{+}}\right)$under the point $A\left(x_{A}, y_{A}\right)$, thus the subsequent function of point $A$ is

$$
g(A)=y_{A^{+}}-y_{A}<0
$$

The phase set $\sum_{N}$ intersects with $\mathrm{x}$-axis at point $B\left(x_{B}, 0\right)$, where $x_{B}=h(1-\alpha(h))$. By the orbit tendency, $y_{H^{-}}(x, B)$ intersects with the impulsive set $\sum_{M}$ at the point $B^{-}\left(x_{B^{-}}, y_{B^{-}}\right)$ which jumps to the point $B^{+}\left(x_{B^{+}}, y_{B^{+}}\right)$. Obviously, the point $B(h(1-\alpha(h)), 0)$ is under the point $B^{+}\left(x_{B^{+}}, y_{B^{+}}\right)$. The subsequent function of the point $B(h(1-\alpha(h)), 0)$ is

$$
g(B)=y_{B^{+}}-y_{B}>0 .
$$

According to the continuity of subsequent function, there must be a point $C$ between point $A$ and $B$, which makes

$$
g(C)=0 \text {. }
$$

(See Figures 2(a) and 2(b).)

(ii) $\sigma(h)>\bar{\sigma}$

If $\sigma(h)>\bar{\sigma}$, then the point $A^{+}\left(x_{A^{+}}, y_{A^{+}}\right)$must be above the point $A\left(x_{A}, y_{A}\right)$. Thus the subsequent function $g(A)>$ 0 . The orbit $y_{H^{-}}\left(x, A^{+}\right)$will intersect with impulsive set $\sum_{M}$ at point $A_{1}^{-}\left(x_{A_{1}^{-}}, y_{A_{1}^{-}}\right)$, then hits phase set $\sum_{N}$ at point $A_{1}^{+}\left(x_{A_{1}^{+}}, y_{A_{1}^{+}}\right)$. Clearly, the point $A_{1}^{-}\left(x_{A_{1}^{-}}, y_{A_{1}^{-}}\right)$is under the point $A^{-}\left(x_{A^{-}}, y_{A^{-}}\right)$. Thus the point $A_{1}^{+}\left(x_{A_{1}^{+}}, y_{A_{1}^{+}}\right)$must be under the point $A^{+}\left(x_{A^{+}}, y_{A^{+}}\right)$. The subsequent function of point $A^{+}\left(x_{A^{+}}, y_{A^{+}}\right)$is

$$
g\left(A^{+}\right)=y_{A_{1}^{+}}-y_{A^{+}}<0 .
$$

So there must be a point $D \in \sum_{N}$, such that $g(D)=0$ (see Figure 2(c)).

Now, the uniqueness of OOPS of system (7) is to be discussed.

Assuming that $P_{1}, P_{2} \in \widehat{Q A}$, then orbit $\widehat{P_{1} P_{1}^{-} P_{1}^{+}}$and $\widehat{P_{2} P_{2}^{-} P_{2}^{+}}$are OOPS, where $y_{P_{1}}<y_{P_{2}}$.

$$
\begin{aligned}
& y_{P_{1}^{+}}=(1-\beta(h)) h y_{P_{1}^{-}}+\sigma(h), \\
& y_{P_{2}^{+}}=(1-\beta(h)) h y_{P_{2}^{-}}+\sigma(h),
\end{aligned}
$$

Assume

$$
\begin{aligned}
\delta_{P_{1} P_{2}}(x)=y_{H^{-}}\left(x, P_{2}\right)-y_{H^{-}}\left(x, P_{1}\right), & \\
x & \in[(1-\beta(h)) h, h],
\end{aligned}
$$


then

$$
\begin{aligned}
& \delta_{P_{1} P_{2}}^{\prime}(x)=y_{H^{-}}^{\prime}\left(x, P_{2}\right)-y_{H^{-}}^{\prime}\left(x, P_{1}\right) \\
& \quad=\frac{\mu x y-l y}{\left(m x-r x^{2}\right) /(K+d x)-q x y} \\
& \quad=\frac{(\mu x-l)(K+d x)}{x}\left[\frac{y P_{2}}{m-r x-q y_{P_{2}}(K+d x)}\right. \\
& \left.-\frac{y P_{1}}{m-r x-q y_{P_{1}}(K+d x)}\right]=\frac{(\mu x-l)(K+d x)}{x} \\
& \cdot \omega(\xi)\left(y_{P_{2}}-y_{P_{1}}\right),
\end{aligned}
$$

where

$$
\omega(y)=\frac{y}{m-r x-q y(K+d x)},
$$

and

$$
\omega^{\prime}(y)=\frac{y(r+d q y)}{[m-r x-q y(K+d x)]^{2}}>0 .
$$

So $\delta_{P_{1} P_{2}}(x)<0, x \in[(1-\alpha) h, h]$; that is to say, $\delta_{P_{1} P_{2}}(x)$ is a decreasing function. For $x \in[(1-\alpha) h, h]$, then $\delta_{P_{1} P_{2}}(h)<$ $\delta_{P_{1} P_{2}}((1-\alpha) h)$.

Thus

$$
\begin{aligned}
\sigma(h)= & y_{P_{2}^{+}}-(1-\beta) y_{P_{2}^{-}}=y_{P_{2}}-(1-\beta) y_{P_{2}^{-}} \\
= & y_{P_{1}}+\delta_{P_{1} P_{2}}((1-\alpha) h) \\
& -(1-\beta)\left(y_{P_{1}^{-}}+\delta_{P_{1} P_{2}}(h)\right) \\
= & y_{P_{1}}-(1-\beta) y_{P_{1}^{-}}+\delta_{P_{1} P_{2}}((1-\alpha) h) \\
& -(1-\beta) \delta_{P_{1} P_{2}}(h)>y_{P_{1}}-(1-\beta) y_{P_{1}^{-}} \\
= & \sigma(h) .
\end{aligned}
$$

which is a contradiction.

When $\sigma(h)>\bar{\sigma}$, then there exists a point $P \in \overline{A A^{+}}$ such that $y_{P^{+}}=y_{P}$. Thus, for any $P^{\prime} \in \overline{B A^{+}}$, the subsequent function of point $P^{\prime}$ is

$$
\begin{aligned}
g\left(P^{\prime}\right) & =y_{P^{\prime+}}-y_{P^{\prime}}=(1-\beta) y_{P^{\prime-}}+\sigma(h)-y_{P^{\prime}} \\
& =(1-\beta) y_{P^{\prime-}}-y_{P^{\prime}}+y_{P}-(1-\beta) y_{P^{-}} \\
& =\left(y_{P}-y_{P^{\prime}}\right)+(1-\beta)\left(y_{P^{\prime-}}-y_{P^{-}}\right) .
\end{aligned}
$$

According to the proof above, we have $\left|y_{P}-y_{P^{\prime}}\right|>\mid y_{P}-$ $y_{P^{\prime-}} \mid$. Thus, if $y_{P^{\prime}}<y_{P}$, then $g\left(P^{\prime}\right)<0$.

If $y_{P^{\prime}}>y_{P}$, then $g\left(P^{\prime}\right)>0$. Thus the uniqueness of OOPS of system (7) in case of $\sigma(h)>\bar{\sigma}$ is proved. The proof is completed.

Theorem 4. If $\max \left\{x^{*}, h_{1}\right\}<h<h_{2}$ holds, then we have two cases. If $h>h_{0}$ holds, then system (7) has no OOPS. If $h<h_{0}$ holds, then system (7) has a unique OOPS, where $h_{0}=\max \{x \mid$ $\left.0<y_{H^{-}}(x, B) \leq h_{2}\right\}$.

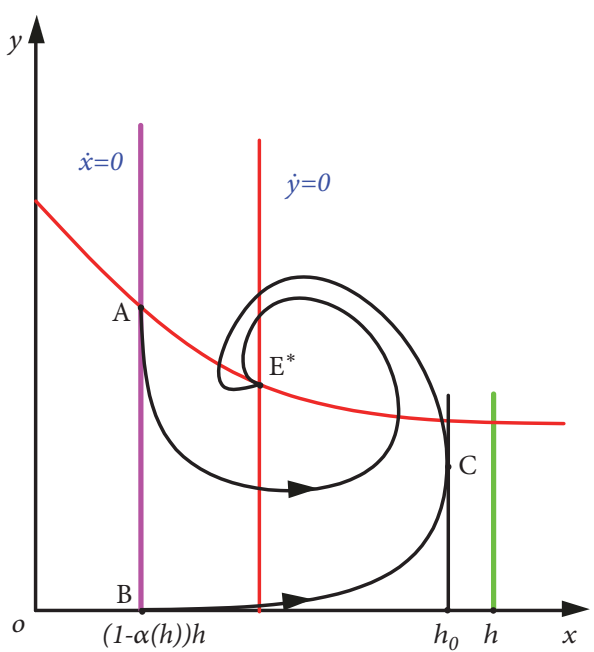

FIgure 3: The existence of the OOPS of system (2) if $h>h_{0}$ in Case (II).

Proof.

Case II $\left(\max \left\{x^{*}, h_{1}<h \leq h_{2}\right\}\right)$. Two cases are discussed according the magnitude of $h$ and $h_{0}$.

(i) If $h>h_{0}$, the trajectory $y_{H^{-}}(x, B)$ tending to point $E^{*}\left(x^{*}, y^{*}\right)$ is based on the global asymptotical stability of $E^{*}\left(x^{*}, y^{*}\right)$. For any $P(x, y) \in \Omega$, the trajectory $y_{H^{-}}(x, P)$ tends to $E^{*}\left(x^{*}, y^{*}\right)$. (See Figure 3.)

(ii) If $h<h_{0}$, the trajectory $y_{H^{-}}(x, B)$ must intersect with the impulsive set $\sum_{M}$ at the point $B^{-}\left(x_{B^{-}}, y_{B^{-}}\right)$, which jumps to the point $B^{+}\left(x_{B^{+}}, y_{B^{+}}\right)$. The subsequent function of point $B\left(x_{B}, y_{B}\right)$ is $g(B)=y_{B^{+}}-y_{B}>0$. Similar to the proof of Case I, when $\sigma \leq \bar{\sigma}$, an OOPS exists in system (7). If $\sigma>\bar{\sigma}$, we also can prove that an OOPS exists in system (7) by same method of Case I. (See Figure 4.)

\section{The Orbital Asymptotical Stability of OOPS of System (7)}

According to the discussion above, a unique OOPS exists in system (7), denoted by $\widehat{P P^{-} P^{+}}$. Then we get the following theorem.

Theorem 5. If $\sigma \leq \bar{\sigma}$, then the OOPS of system (7) is orbitally asymptotically stable and globally attractive to the point $E^{*}$.

Proof. We choose arbitrary point $A_{0}$ on the phase set $\sum_{N}$. If $A_{0} \in N / \overline{A P}$, then after several pulse effects the trajectory will jump to the segment $\overline{A P}$. Thus we assume that $A_{0} \in \overline{A P}$; the trajectory $y_{H^{-}}\left(x, A_{0}\right)$ will hit the impulsive set $\sum_{M}$ at point $A_{1}^{-}$, which jumps to the point $A_{1}^{+}$. The trajectory $y_{H^{-}}\left(x, A_{1}^{+}\right)$ will intersect with impulsive set $\sum_{M}$ at point $A_{2}^{-}$and then jumps to the point $A_{2}^{+}$. Repeat the process above; we get a point sequences $\left\{A_{k}^{+}\right\}$, where $k=1,2,3, \cdots$ such that

$$
y_{A}>y_{A_{0}}>y_{A_{1}}^{+}>\cdots>y_{A_{k}}^{+}>\cdots \geq y_{P} .
$$




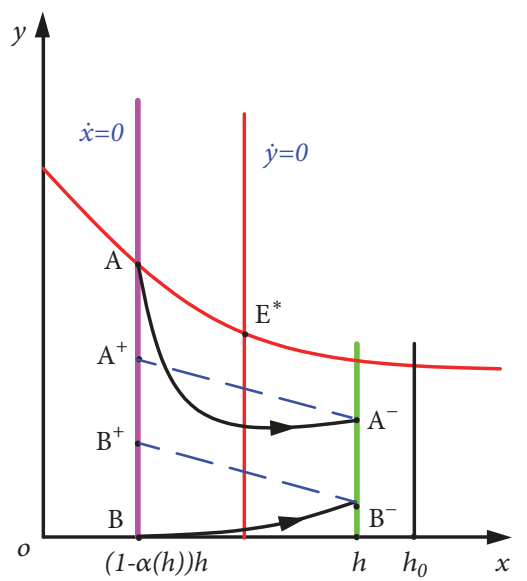

(a)

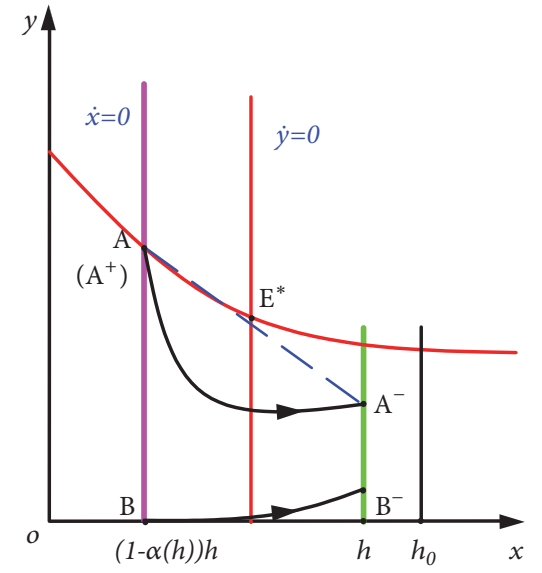

(b)

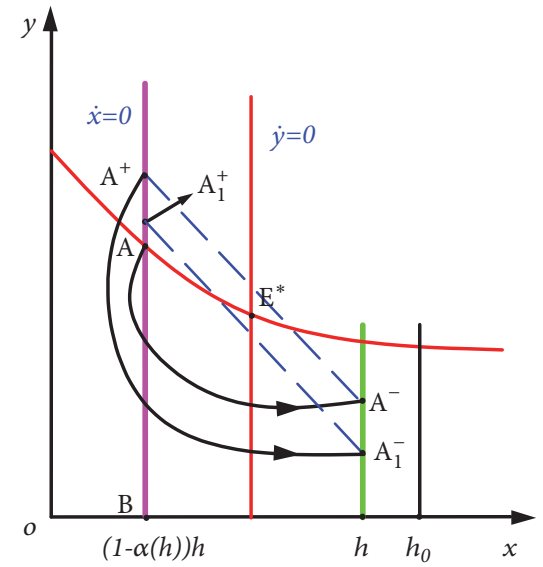

(c)

Figure 4: The existence of the OOPS of system (7) if $h<h_{0}$ in Case (II).

The sequence $A_{k}^{+} \|_{k=0,1,2, \ldots}$ is a monotonic decreasing sequence with lower bound $y_{P}$. According to the monotonic bounded theorem, there must exist a limit $y_{P^{\prime}}$ such that $\lim _{k \rightarrow \infty} y_{A_{k}^{+}}=y_{P^{\prime}}$, which means that

$$
\begin{aligned}
g\left(P^{\prime}\right) & =g\left(\lim _{k \rightarrow \infty} y_{A_{k}^{+}}\right)=\lim _{k \rightarrow \infty} g\left(y_{A_{k}^{+}}\right) \\
& =\lim _{k \rightarrow \infty}\left(y_{A_{k+1}^{+}}-y_{A_{k}^{+}}\right)=0 .
\end{aligned}
$$

Since $g(A)=0$, if and only if $A=P$, then $P^{\prime}=P$. That is to say $\lim _{k \rightarrow \infty} y_{A_{k}^{+}}=y_{P}$.

Similarly, we can use the above method to get an increasing point sequences $B_{k}^{+} \|_{k=0,1,2, \ldots}$ such that

$$
y_{B}<y_{B_{0}}<y_{B_{1}}^{+}<\cdots<y_{B_{k}}^{+}<\cdots \leq y_{P}
$$

There must exist a limit $y_{P^{\prime}}$ such that $\lim _{k \rightarrow \infty} y_{B_{k}^{+}}=y_{P^{\prime}}$, which means that

$$
\begin{aligned}
g\left(P^{\prime}\right) & =g\left(\lim _{k \rightarrow \infty} y_{B_{k}^{+}}\right)=\lim _{k \rightarrow \infty} g\left(y_{B_{k}^{+}}\right) \\
& =\lim _{k \rightarrow \infty}\left(y_{B_{k+1}^{+}}-y_{B_{k}^{+}}\right)=0 .
\end{aligned}
$$

Since $g(B)=0$, if and only if $B=P$, then $P^{\prime}=P$. That is to say, $\lim _{k \rightarrow \infty} y_{B_{k}^{+}}=y_{P}$. By the arbitrariness of the point $A_{0}$ and $B_{0}$, one has

$$
\lim _{k \rightarrow \infty} y_{A_{k}^{+}}=\lim _{k \rightarrow \infty} y_{B_{k}^{+}}=y_{P} .
$$

Thus the OOPS of system (7) is orbitally asymptotically stable and globally attractive (see Figure 5).

Theorem 6. If $\sigma>\bar{\sigma}$ and $\gamma_{1}\left[m-r \eta_{0}-q \gamma_{0}\left(K+d \eta_{0}\right)\right](1-$ $\beta)\left(K+d \eta_{1}\right) / \gamma_{0}\left(m-r \eta_{1}-q \gamma_{1}\right)\left(K+d \eta_{0}\right)<1$, then the OOPS of system (7) is orbitally asymptotically stable.

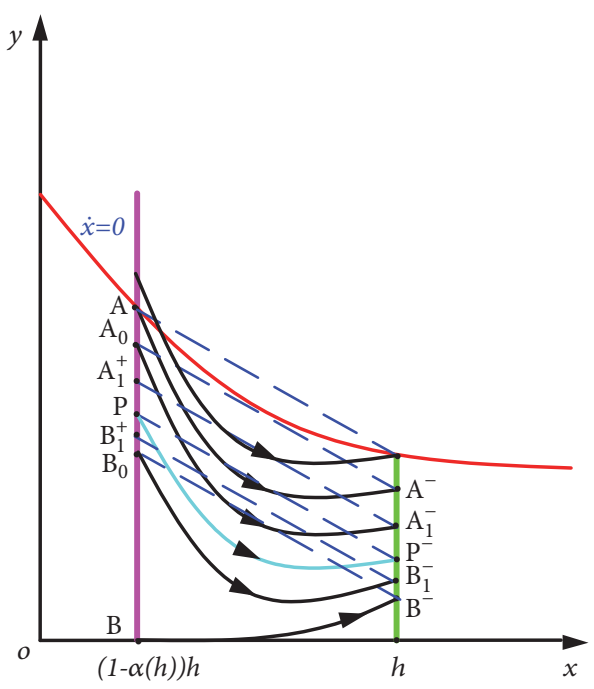

FIGURE 5: The orbitally asymptotically stability of the OOPS of system (7).

Proof. Let $x=\eta(t), y=\gamma(t)$ is a T-periodic orbit of system (7) and $\eta_{0}=\eta(0), \gamma_{0}=\gamma(0), \eta_{1}=\eta(T), \gamma_{1}=\gamma(T), \eta_{1}^{+}=$ $\eta\left(T^{+}\right), \gamma_{1}^{+}=\gamma\left(T^{+}\right)$, then

$$
\begin{aligned}
& \eta_{1}^{+}=\eta_{0}=(1-\alpha) h, \\
& \gamma_{1}^{+}=\gamma_{0}=(1-\beta) \gamma_{1}+\sigma .
\end{aligned}
$$

Let

$$
\begin{aligned}
& P(x, y)=\frac{m x-r x^{2}}{K+d x}-q x y, \\
& Q(x, y)=\mu x y-l y, \\
& \zeta(x, y)=-\alpha x \\
& \xi(x, y)=-\beta y+\sigma .
\end{aligned}
$$


Then

$$
\begin{aligned}
\frac{\partial \zeta}{\partial x} & =-\alpha, \\
\frac{\partial \xi}{\partial x} & =-\beta, \\
\frac{\partial \zeta}{\partial y} & =0, \\
\frac{\partial \xi}{\partial y} & =-\beta, \\
\frac{\partial \varphi}{\partial x} & =1, \\
\frac{\partial \varphi}{\partial y} & =0 \\
\Delta{ }_{1} & =\frac{P+((\partial \xi / \partial y)(\partial \varphi / \partial x)-(\partial \xi / \partial x)(\partial \varphi / \partial y)+\partial \varphi / \partial x)+Q+((\partial \zeta / \partial x)(\partial \varphi / \partial y)-(\partial \zeta / \partial y)(\partial \varphi / \partial x)+\partial \varphi / \partial y)}{P(\partial \varphi /(\partial x+Q(\partial \varphi / \partial y)))} \\
& =\frac{P\left(\eta_{1}^{+}, \gamma_{1}^{+}\right)(-\beta-0+1)+Q\left(\eta_{1}^{+}, \gamma_{1}^{+}\right)(-\alpha \times 0-r \times 0+0)}{P\left(\eta_{1}, \gamma_{1}\right) \times 1+Q\left(\eta_{1}, \gamma_{1}\right) \times 0}=\frac{\eta_{0}\left[\left(m-r \eta_{0}\right) /\left(K+d \eta_{0}\right)-q \gamma_{0}\right](1-\beta)}{\eta_{1}\left[\left(m-r \eta_{1}\right) /\left(K+d \eta_{1}\right)-q \gamma_{1}\right]} .
\end{aligned}
$$

and

$$
\begin{aligned}
\int_{0}^{T} & \left(\frac{\partial P}{\partial x}+\frac{\partial Q}{\partial y}\right) d t=\int_{0}^{T}\left[\frac{m-2 r x}{K+d x}-\frac{d\left(m x-r x^{2}\right)}{(K+d x)^{2}}\right. \\
- & q y+\mu x-l] d t=\int_{0}^{T}\left[\frac{m-r x}{K+d x}-\frac{r x}{K+d x}\right. \\
& \left.-\frac{d\left(m x-r x^{2}\right)}{(K+d x)^{2}}-q y+\mu x-l\right] d t \\
& =\int_{0}^{T}\left[\frac{\dot{x}}{x(t)}+\frac{\dot{y}}{y(t)}\right]-\left[\int_{0}^{T} \frac{r x}{K+d x}\right. \\
& \left.+\frac{d\left(m x-r x^{2}\right)}{(K+d x)^{2}}\right] d t=\ln \frac{x(T) y(T)}{x(0) y(0)} \\
& -\int_{0}^{T} \frac{r x(K+d x)+d\left(m x-r x^{2}\right)}{(K+d x)^{2}} d t \\
& =\ln \frac{x(T) y(T)}{x(0) y(0)}-\int_{0}^{T} \frac{d m x+r K x}{(K+d x)^{2}} d t . \\
\mu_{2} & =\Delta_{1} \int_{0}^{T}\left(\frac{\partial P}{\partial x}+\frac{\partial Q}{\partial y}\right) d t \\
& =\frac{\left[m \eta_{0}-r \eta_{0}^{2}-q \eta_{0} \gamma_{0}\left(K+d \eta_{0}\right)\right](1-\beta)}{K+d \eta_{0}}
\end{aligned}
$$

$$
\begin{aligned}
& \cdot \frac{K+d \eta_{1}}{m \eta_{1}-r \eta_{1}^{2}-q \eta_{1} \gamma_{1}} \frac{\eta_{1} \gamma_{1}}{\eta_{0} \gamma_{0}} \exp \int_{0}^{T} \frac{-d m x-r K x}{(K+d x)^{2}} \\
& =\frac{\gamma_{1}\left[m-r \eta_{0}-q \gamma_{0}\left(K+d \eta_{0}\right)\right](1-\beta)\left(K+d \eta_{1}\right)}{\gamma_{0}\left(m-r \eta_{1}-q \gamma_{1}\right)\left(K+d \eta_{0}\right)} \\
& \cdot \exp \int_{0}^{T} \frac{-d m x-r K x}{(K+d x)^{2}} .
\end{aligned}
$$

Thus, when $\sigma>\bar{\sigma}$ and $\gamma_{1}\left[m-r \eta_{0}-q \gamma_{0}\left(K+d \eta_{0}\right)\right](1-\beta)(K+$ $\left.d \eta_{1}\right) / \gamma_{0}\left(m-r \eta_{1}-q \gamma_{1}\right)\left(K+d \eta_{0}\right)<1,\left|\mu_{2}\right|<1$. Therefore, the OOPS is orbitally asymptotically stable.

\section{Numerical Simulations and Optimization of Pest Control Level}

5.1. Numerical Simulations. In this section, the feasibility of our conclusions is verified by an example. Let $m=1, r=$ $0.3, K=2, d=0.5, q=0.6, \mu=0.5$, and $l=0.4$. By calculation, the equilibrium point $E^{*}$ of system (7) is $E^{*}(0.8,0.528)$. Parameter values are taken into system (7), then

$$
\begin{aligned}
& \frac{d x}{d t}=\frac{x(t)-0.3 x(t)^{2}}{2+0.5 x(t)}-0.6 x(t) y(t), \\
& \frac{d y}{d t}=0.5 x(t) y(t)-0.4 y(t),
\end{aligned}
$$

$$
x<h,
$$




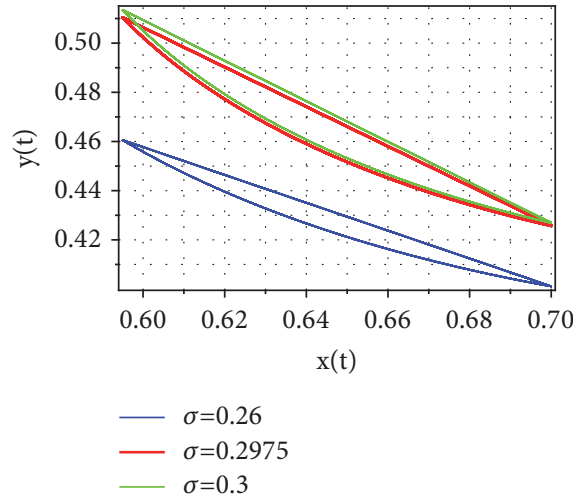

(a)

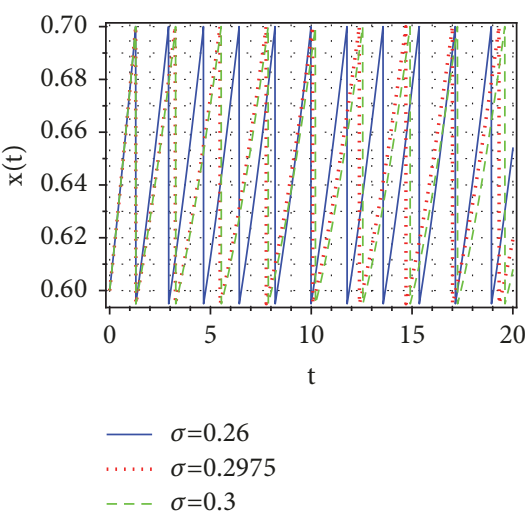

(b)

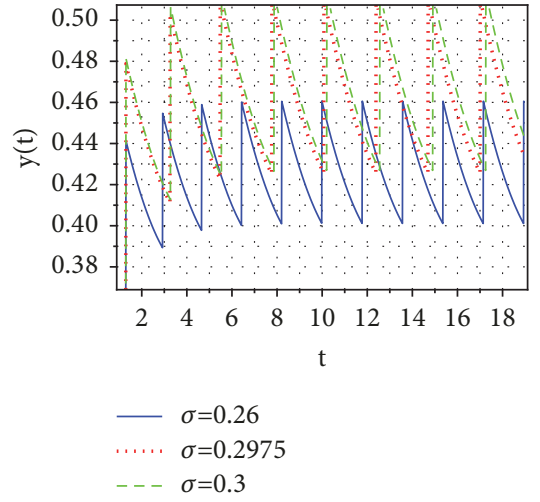

(c)

Figure 6: Numerical simulations in case $0<h_{1}<h<\min \left\{x^{*}, h_{2}\right\}$. (a) Phase portrait of $x(t)$ and $y(t)$ on $h=0.7$. (b) Time series of $x(t)$. (c) Time series of $y(t)$.

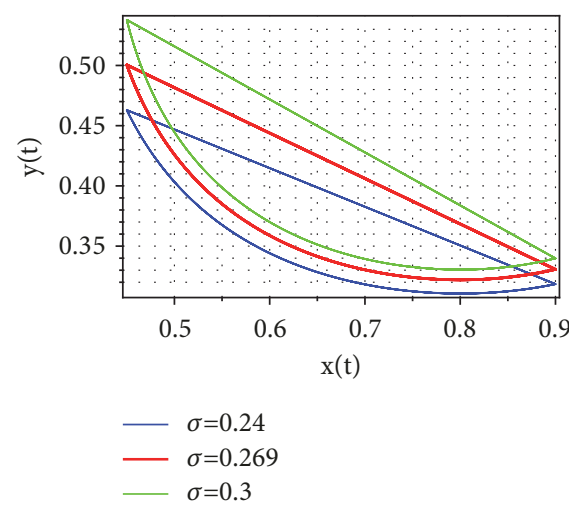

(a)

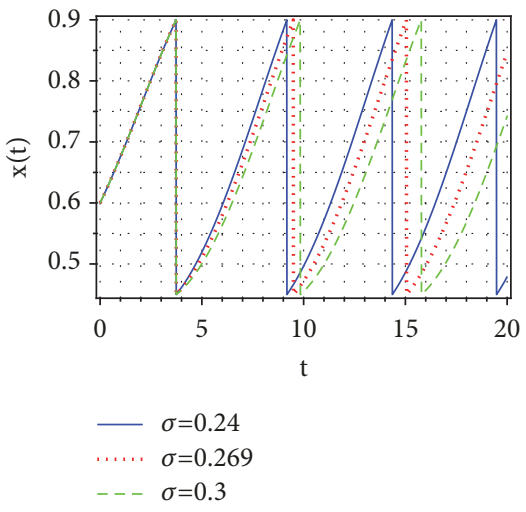

(b)

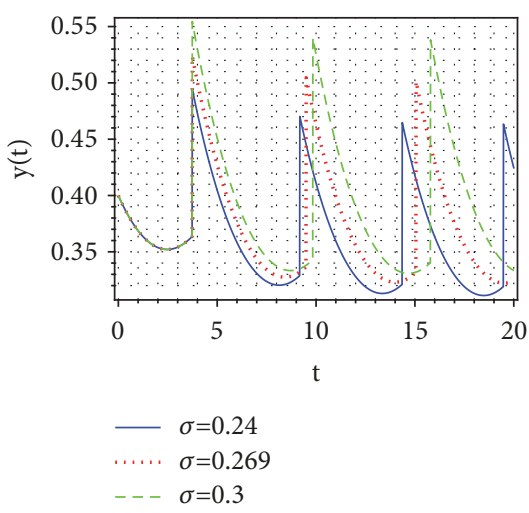

(c)

Figure 7: Numerical simulations in case $0<\max \left\{x^{*}, h_{1}\right\}<h<h_{0}<h_{2}$. (a) Phase portrait of $x(t)$ and $y(t)$ on $h=0.9$. (b) Time series of $x(t)$. (c) Time series of $y(t)$.

$$
\begin{aligned}
& \Delta x(t)=-\alpha(x) x(t), \\
& \Delta y(t)=-\beta(x) y(t)+\sigma(x),
\end{aligned}
$$

$$
x=h, y \leq \bar{y}_{h}
$$

Let $h=0.7$ satisfy the condition $0<h_{1}<h<\min \left\{x^{*}, h_{2}\right\}$ and the initial value be $(0.4,0.4)$. Let $\sigma_{\max }=1.5, \delta_{\text {min }}=0.1$, $\alpha_{\max }=1.75, \beta_{\max }=2, h_{1}=0$, and $h_{2}=2$. A directed calculation yields that $\alpha_{0.7}=0.15, \beta_{0.7}=0.5$, and $\overline{\sigma_{0.7}}=$ 0.2975 . Let $\sigma=0.26, \sigma=\overline{\sigma_{0.7}}=0.2975$, and $\sigma=0.3$. Figures 6(a), 6(b), and 6(c) show that a unique and asymptotically stable OOPS exists in system (7).

Let $h=0.9$ satisfy the condition $0<\max \left\{x^{*}, h_{1}\right\}<h<$ $h_{0}<h_{2}$ and the initial value be $(0.5,1)$. A directed calculation yields that $\alpha_{0.9}=0.5, \beta_{0.9}=0.3$, and $\overline{\sigma_{0.9}}=0.269$. Let $\sigma=0.24, \sigma=\overline{\sigma_{0.9}}=0.269$, and $\sigma=0.3$. Figures 7(a), 7(b), and 7 (c) show that system (7) has a unique and asymptotically stable OOPS.

For the case of $0<\max \left\{x^{*}, h_{1}\right\}<h_{0}<h \leq h_{2}$, for example, $h=1.5$ and the orbit of system (7) starts from
$(0.5,1)$, we get $\alpha_{1.5}=0.5, \beta_{0.9}=0.3$ and $\sigma_{1.5}=0.24$ by calculation. Figures $8(\mathrm{a}), 8(\mathrm{~b})$, and $8(\mathrm{c})$ show that system (7) has no OOPS.

5.2. Determination and Optimization of Pest Control Level. The goal to investigate the existence of OOPS of system (7) lies in that it can obtain the possibility of determining the frequency of releasing predators and spraying pesticides, which makes the density of pest below the damage level. Although the density of prey is inaccurate or biased, the system will eventually undergo periodic changes under the effective control. The following problems are considered to determine the optimal frequency for releasing predators and chemical controls.

Assuming that unit cost of releasing predator is denoted by $\iota_{1}$ and the unit cost of spraying pesticides is denoted by $\iota_{2}$, which include the price of chemical agent and the price of the damage to environment. Our goal is to reduce the unit cost in this process. In one period, the total cost is denoted by $F_{l}$, which is a function about $\alpha(h)$ (i.e., chemical control strength) and $\sigma(h)$ (i.e., yield of releases of predator). 


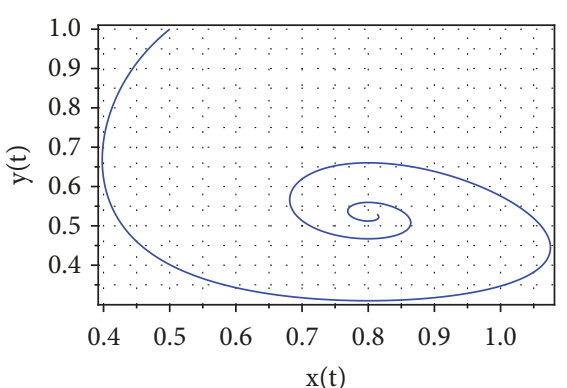

$-\sigma=0.24$

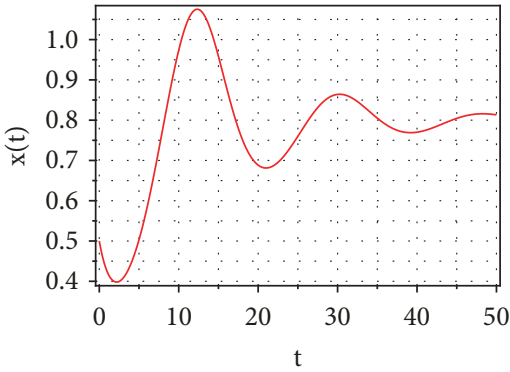

$-\sigma=0.24$

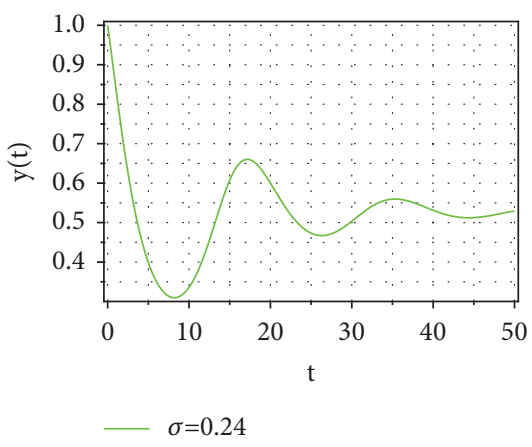

(c)

Figure 8: Numerical simulations in case $<\max \left\{x^{*}, h_{1}\right\}<h_{0}<h \leq h_{2}$. (a) Phase portrait of $x(t)$ and $y(t)$ on $h=1.5$. (b) Time series of $x(t)$. (c) Time series of $y(t)$.

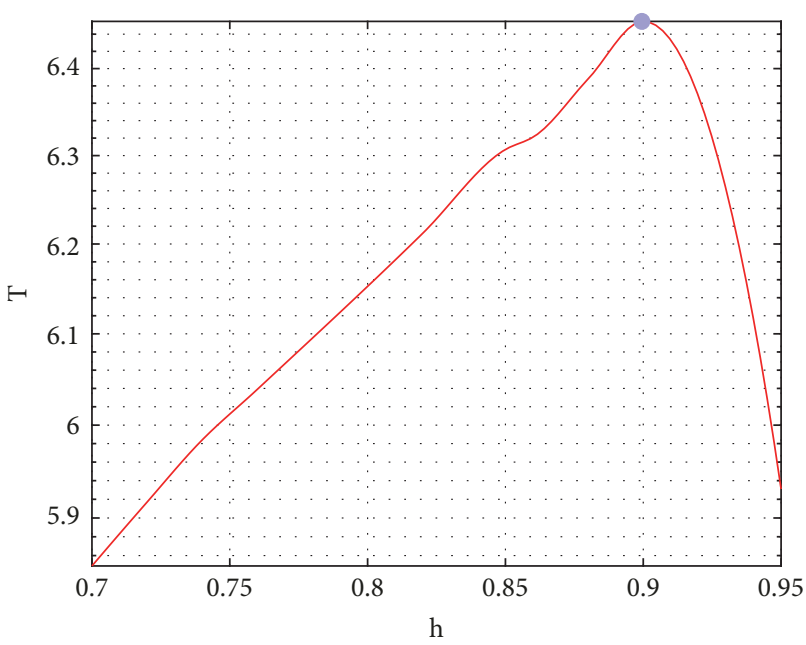

(a)

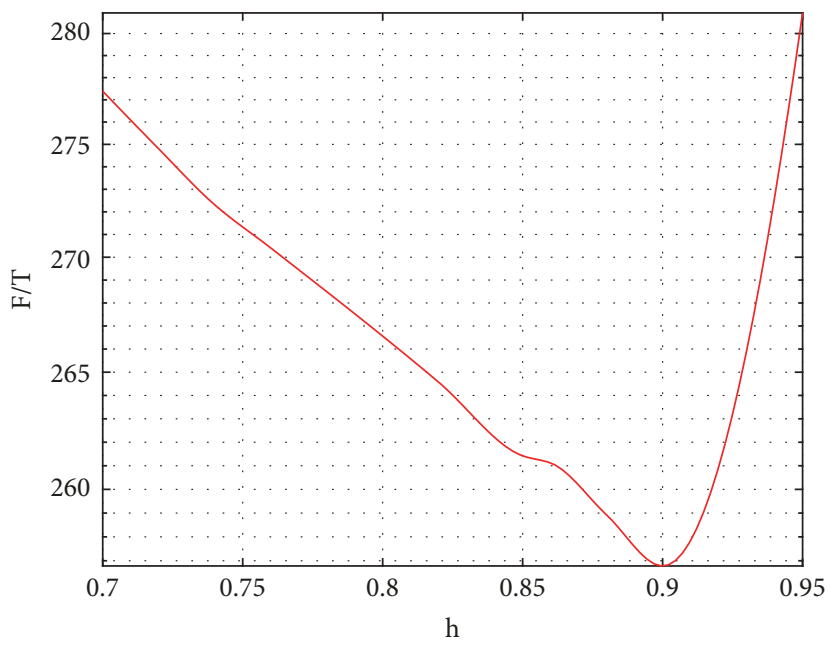

(b)

Figure 9: The variety in the period $T$ and the profit per unit time $F / T$ on the threshold $h$. (a) The variety in the period $T$ on the threshold $h$. (b) The profits per unit time $F / T$ on the threshold $h$.

Then $F_{l}(h)=\iota_{1} \sigma(h)+\iota_{2} \alpha(h)$. So the optimization model is formulated as

$$
\begin{array}{ll}
\max & \frac{F_{\iota}(h)}{T(h)} \\
\text { s.t. } & h_{1} \leq h \leq h_{2}
\end{array}
$$

The optimization problem is solved to yield the optimal pest level $h^{*}$, which the optimal release rate of predator is $\sigma^{*}=\sigma_{h^{*}}$, the optimal strength of chemical control is $\alpha^{*}=$ $\alpha_{h^{*}}$, and the optimal impulse period of chemical control is $T^{*}=T\left(\sigma^{*}, \alpha^{*}\right)$. However, the optimum pest control level $h^{*}$ is dependent on the ratio of $\omega \triangleq \iota_{2} / \iota_{1}$. The impulse period $T$ varies with the threshold $h$, as shown in Figure 9(a). And Figure 9(b) shows the variation of cost per unit time $F / T$ and the period $T$ with the pest control level $h$, where $\iota_{1}=1000$, $\iota_{2}=1000$, i.e., $\omega=1$. The optimal pest level is $h^{*}=0.9$, the optimal strength of chemical control is $\alpha_{h^{*}}=0.788$, and the optimal release rate of predator is $\sigma_{h^{*}}=0.87$. It is important to note that the optimum economic threshold $h$ is dependent on $\omega$, as is illustrated in Figure 10.

\section{Conclusion}

A Smith prey-predator system with linear feedback control for integrated pest management is investigated in this paper. Integrated control strategy is more practical which can maximize the protection of the ecological environment and reduce the cost of pest management. First, the method of subsequent function and differential equation geometry theory are used to prove the existence, uniqueness, and stability of the OOPS of system (7). Second, a specific example is given to verify the conclusion of the impulsive strategy. Last, an optimized problem is formulated and the minimized total cost in pest control is obtained. However, the optimized results have some deviations which need to be further improved.

\section{Data Availability}

We agree to share the data underlying the findings of the manuscript. Data sharing allows researchers to verify the results of an article, replicate the analysis, and conduct secondary analyses. 

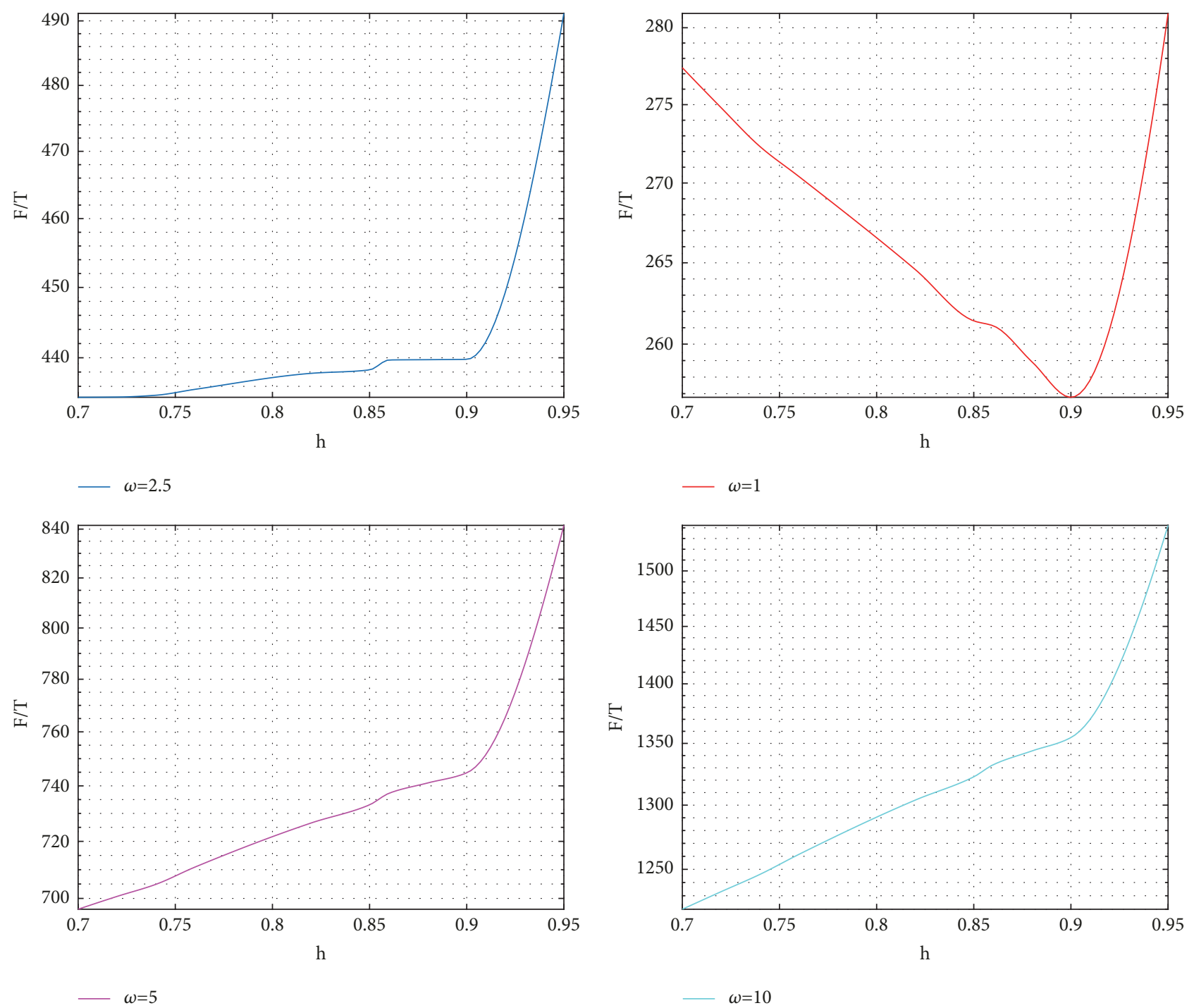

FIgURE 10: The change in the cost per unit time $F / T$ on the control level $h$ for $\iota_{2} / \iota_{1}=2.5,1,5,10$.

\section{Conflicts of Interest}

The authors declare that they have no conflicts of interest.

\section{Acknowledgments}

This work was supported by the National Natural Science Foundation of China (11371230 and 11501331), the SDUST Research Fund (2014TDJH102), Shandong Provincial Natural Science Foundation, China (ZR2015AQ001 and BS2015SF002), Joint Innovative Center for Safe and Effective Mining Technology and Equipment of Coal Resources, the Open Foundation of the Key Laboratory of Jiangxi Province for Numerical Simulation and Emulation Techniques, Gannan Normal University, China, and SDUST Innovation Fund for Graduate Students (no. SDKDYC170225).

\section{References}

[1] M. Huang and X. Song, "Periodic Solutions and Homoclinic Bifurcations of Two Predator-Prey Systems with Nonmonotonic
Functional Response and Impulsive Harvesting," Journal of Applied Mathematics, vol. 2014, Article ID 803764, 11 pages, 2014.

[2] C. J. Wei and L. S. Chen, "Periodic solution and heteroclinic bifurcation in a predator-prey system with Allee effect and impulsive harvesting," Nonlinear Dynamics, vol. 76, no. 2, pp. 1109-1117, 2014.

[3] Y. Li, H. Cheng, and Y. Wang, "A Lycaon pictus impulsive state feedback control model with Allee effect and continuous time delay," Advances in Difference Equations, Paper No. 367, 17 pages, 2018.

[4] B. Liu, Y. Tian, and B. Kang, "Dynamics on a Holling II predator-prey model with state-dependent impulsive control," International Journal of Biomathematics, vol. 5 , no. 3, Article ID 1260006, pp. 93-110, 2012.

[5] M. Zhang, G. Song, and L. Chen, "A state feedback impulse model for computer worm control," Nonlinear Dynamics, vol. 85, no. 3, pp. 1561-1569, 2016.

[6] X. Z. Meng, S. N. Zhao, T. Feng, and T. H. Zhang, "Dynamics of a novel nonlinear stochastic SIS epidemic model with double 
epidemic hypothesis," Journal of Mathematical Analysis and Applications, vol. 433, no. 1, pp. 227-242, 2016.

[7] T. Zhang, T. Zhang, and X. Meng, "Stability analysis of a chemostat model with maintenance energy," Applied Mathematics Letters, vol. 68, pp. 1-7, 2017.

[8] Huidong Cheng, Tongqian Zhang, and Fang Wang, "Existence and Attractiveness of Order One Periodic Solution of a Holling I Predator-Prey Model," Abstract and Applied Analysis, vol. 2012, Article ID 126018, 18 pages, 2012.

[9] H. Liu and H. Cheng, "Dynamic analysis of a prey-predator model with state-dependent control strategy and square root response function," Advances in Difference Equations, Paper No. 63, 13 pages, 2018.

[10] K. B. Sun, T. H. Zhang, and Y. Tian, "Theoretical study and control optimization of an integrated pest management predator-prey model with power growth rate," Mathematical Biosciences, vol. 279, pp. 13-26, 2016.

[11] G. Liu, X. Wang, X. Meng, and S. Gao, "Extinction and Persistence in Mean of a Novel Delay Impulsive Stochastic Infected Predator-Prey System with Jumps," Complexity, vol. 2017, Article ID 1950970, 15 pages, 2017.

[12] H. Zhang, J. Jiao, and L. Chen, "Pest management through continuous and impulsive control strategies," BioSystems, vol. 90, no. 2, pp. 350-361, 2007.

[13] F. Wang, B. Chen, Y. Sun, and C. Lin, "Finite time control of switched stochastic nonlinear systems," Fuzzy Sets and Systems, 2018.

[14] G. Pang and L. Chen, "Periodic solution of the system with impulsive state feedback control," Nonlinear Dynamics, vol. 78, no. 1, pp. 743-753, 2014.

[15] W. Lv, F. Wang, and Y. Li, "Adaptive finite-time tracking control for nonlinear systems with unmodeled dynamics using neural networks," Advances in Difference Equations, Paper No. 159, 17 pages, 2018.

[16] T. Zhang, W. Ma, and X. Meng, "Global dynamics of a delayed chemostat model with harvest by impulsive flocculant input," Advances in Difference Equations, Paper No. 115, 17 pages, 2017.

[17] W. Lv and F. Wang, "Adaptive tracking control for a class of uncertain nonlinear systems with infinite number of actuator failures using neural networks," Advances in Difference Equations, Paper No. 374, 16 pages, 2017.

[18] F. Liu, "Continuity and approximate differentiability of multisublinear fractional maximal functions," Mathematical Inequalities \& Applications, vol. 21, no. 1, pp. 25-40, 2018.

[19] F. Liu, Q. Xue, and K. Yabuta, "Rough maximal singular integral and maximal operators supported by subvarieties on Triebel-Lizorkin spaces," Nonlinear Analysis. Theory, Methods \& Applications. An International Multidisciplinary Journal, vol. 171, pp. 41-72, 2018.

[20] Y. Li, H. Cheng, J. Wang, and Y. Wang, "Dynamic analysis of unilateral diffusion Gompertz model with impulsive control strategy," Advances in Difference Equations, Paper No. 32, 14 pages, 2018.

[21] T. Zhang, X. Liu, X. Meng, and T. Zhang, "Spatio-temporal dynamics near the steady state of a planktonic system," Computers \& Mathematics with Applications, vol. 75, no. 12, pp. 44904504, 2018.

[22] F. Wang, B. Chen, C. Lin, J. Zhang, and X. Meng, "Adaptive Neural Network Finite-Time Output Feedback Control of Quantized Nonlinear Systems," IEEE Transactions on Cybernetics, vol. 48, no. 6, pp. 1839-1848, 2017.
[23] A. Miao, T. Zhang, J. Zhang, and C. Wang, "Dynamics of a stochastic SIR model with both horizontal and vertical transmission," Journal of Applied Analysis and Computation, vol. 8, no. 4, pp. 1108-1121, 2018.

[24] X. Fan, Y. Song, and W. Zhao, "Modeling Cell-to-Cell Spread of HIV-1 with Nonlocal Infections," Complexity, vol. 2018, pp. 1-10, 2018.

[25] Z. Zhao, L. Pang, and X. Song, "Optimal control of phytoplankton-fish model with the impulsive feedback control," Nonlinear Dynamics, vol. 88, no. 3, pp. 2003-2011, 2017.

[26] H. Guo, L. Chen, and X. Song, "Dynamical properties of a kind of SIR model with cnstant vaccination rate and impulsive state feedback control," International Journal of Biomathematics, vol. 10, no. 7, 1750093, 21 pages, 2017.

[27] G. Z. Zeng, L. S. Chen, and J. F. Chen, "Persistence and periodic orbits for two-species nonautonomous diffusion Lotka-Volterra models," Mathematical and Computer Modelling, vol. 20, no. 12, pp. 69-80, 1994.

[28] F. Liu, "A note on Marcinkiewicz integrals associated to surfaces of revolution," Journal of the Australian Mathematical Society, vol. 104, no. 3, pp. 380-420, 2018.

[29] M. Chi and W. Zhao, "Dynamical analysis of multi-nutrient and single microorganism chemostat model in a polluted environment," Advances in Difference Equations, Paper No. 120, 16 pages, 2018.

[30] M. Huang, X. Song, and J. Li, "Modelling and analysis of impulsive releases of sterile mosquitoes," Journal of Biological Dynamics, vol. 11, no. 1, pp. 147-171, 2017.

[31] A. Miao, J. Zhang, T. Zhang, and P. Aruna, “Threshold dynamics of a stochastic SIR model with vertical transmission and vaccination," Computational and Mathematical Methods in Medicine, vol. 2017, Article ID 4820183, 10 pages, 2017.

[32] F. Liu and H. Wu, "Singular integrals related to homogeneous mappings in Triebel-Lizorkin spaces," Journal of Mathematical Inequalities, vol. 11, no. 4, pp. 1075-1097, 2017.

[33] Y. Song, A. Miao, T. Zhang, X. Wang, and J. Liu, "Extinction and persistence of a stochastic SIRS epidemic model with saturated incidence rate and transfer from infectious to susceptible," Advances in Difference Equations, Paper No. 293, 11 pages, 2018.

[34] Y. Li and X. Liu, "H-index for nonlinear stochastic systems with state and input dependent noises," International Journal of Fuzzy Systems, vol. 20, no. 3, pp. 759-768, 2018.

[35] X. Meng and L. Zhang, "Evolutionary dynamics in a LotkaVolterra competition model with impulsive periodic disturbance," Mathematical Methods in the Applied Sciences, vol. 39, no. 2, pp. 177-188, 2016.

[36] H. Cheng and T. Zhang, "A new predator-prey model with a profitless delay of digestion and impulsive perturbation on the prey," Applied Mathematics and Computation, vol. 217, no. 22, pp. 9198-9208, 2011.

[37] Y. Li, W. Zhang, and X.-K. Liu, "H-index for discrete-time stochastic systems with markovian jump and multiplicative noise," Automatica, vol. 90, pp. 286-293, 2018.

[38] J. Wang, H. Cheng, X. Meng, and B. G. Pradeep, "Geometrical analysis and control optimization of a predator-prey model with multi state-dependent impulse," Advances in Difference Equations, Paper No. 252, 17 pages, 2017.

[39] Q. Liu, L. Huang, and L. Chen, "A pest management model with state feedback control," Advances in Difference Equations, vol. 2016, no. 1, 292 pages, 2016. 
[40] T. Zhang, W. Ma, X. Meng, and T. Zhang, "Periodic solution of a prey-predator model with nonlinear state feedback control," Applied Mathematics and Computation, vol. 266, pp. 95-107, 2015.

[41] L. Chen, "Pest control and geometric theory of semi-continuous dynamical system," Journal of Beihua University, vol. 12, no. 1, 2011.

[42] J. Wang, H. Cheng, H. Liu, and Y. Wang, "Periodic solution and control optimization of a prey-predator model with two types of harvesting," Advances in Difference Equations, Paper No. 41, 14 pages, 2018.

[43] H. Cheng, F. Wang, and T. Zhang, "Multi-state dependent impulsive control for pest management," Journal of Applied Mathematics, vol. 2012, Article ID 381503, 25 pages, 2012.

[44] J. Wang, H. Cheng, Y. Li, and X. Zhang, "The geometrical analysis of a predator-prey model with multi-state dependent impulses," Journal of Applied Analysis and Computation, vol. 8, no. 2, pp. 427-442, 2018.

[45] L. Nie, Z. Teng, L. Hu, and J. Peng, "The dynamics of a LotkaVolterra predator-prey model with state dependent impulsive harvest for predator," BioSystems, vol. 98, no. 2, pp. 67-72, 2009.

[46] Y. Tian, T. Zhang, and K. Sun, "Dynamics analysis of a pest management prey-predator model by means of interval state monitoring and control," Nonlinear Analysis: Hybrid Systems, vol. 23, pp. 122-141, 2017.

[47] L. Zhao, L. Chen, and Q. Zhang, "The geometrical analysis of a predator-prey model with two state impulses," Mathematical Biosciences, vol. 238, no. 2, pp. 55-64, 2012.

[48] T. Q. Zhang, X. Z. Meng, R. Liu, and T. H. Zhang, "Periodic solution of a pest management Gompertz model with impulsive state feedback control," Nonlinear Dynamics, vol. 78, no. 2, pp. 921-938, 2014.

[49] F. E. Smith, "Population dynamics in Daphnia magna and a new model for population growth," Ecology, vol. 44, no. 4, pp. 651663, 1963. 


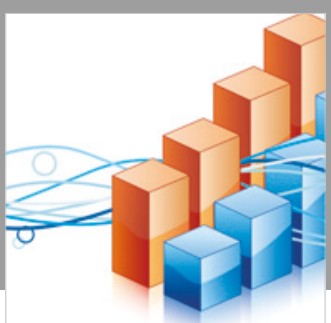

Advances in

Operations Research

\section{-n-m}
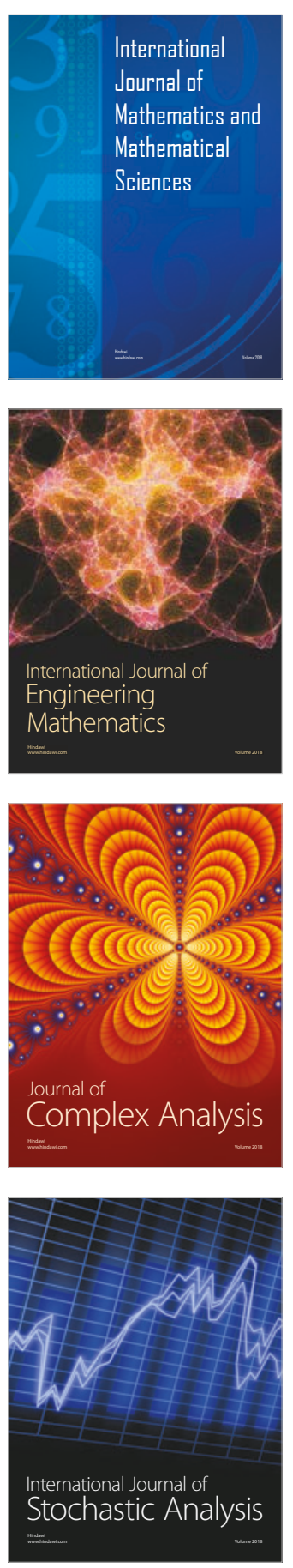
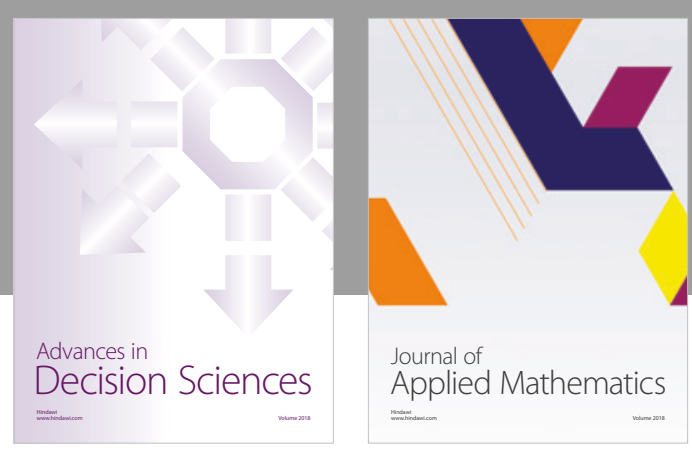

Journal of

Applied Mathematics
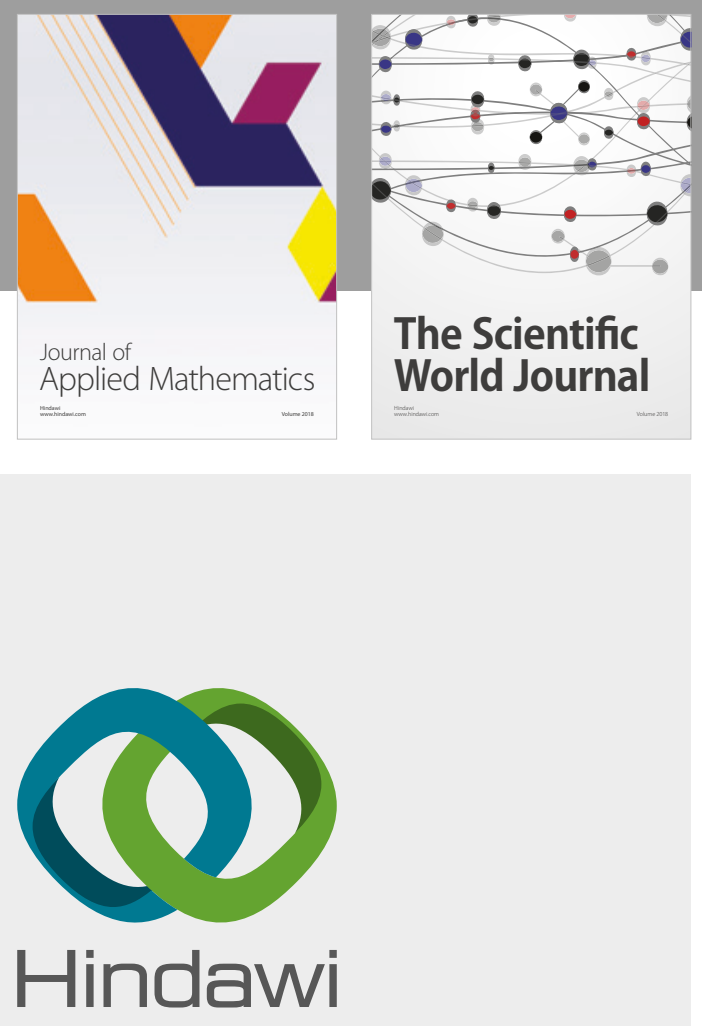

Submit your manuscripts at

www.hindawi.com

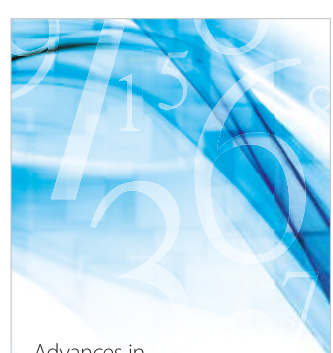

Advances in
Numerical Analysis
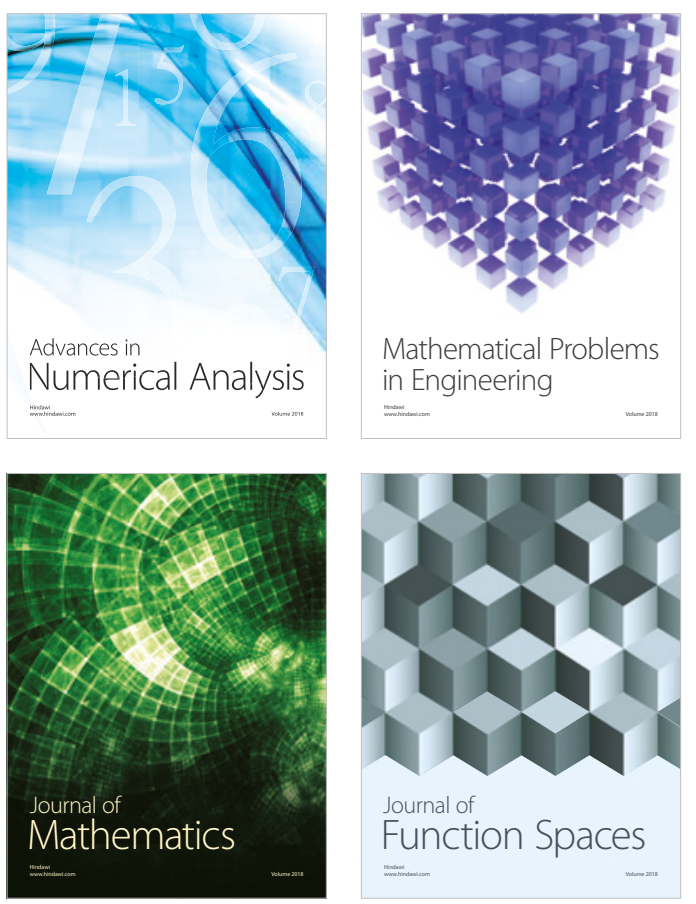

Mathematical Problems in Engineering

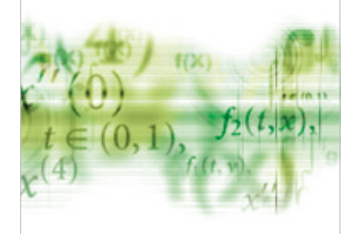

International Journal of

Differential Equations

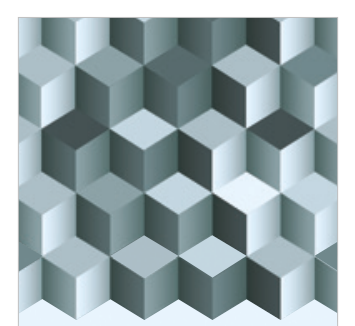

Journal of

Function Spaces

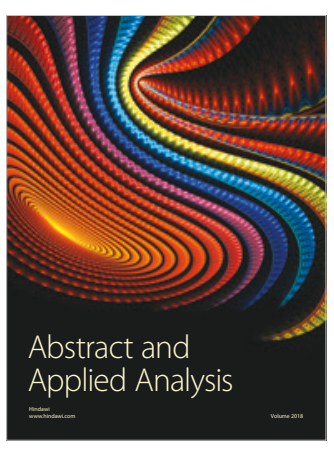

The Scientific

World Journal

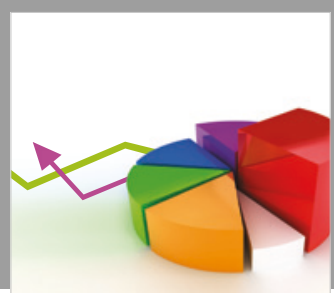

Journal of

Probability and Statistics
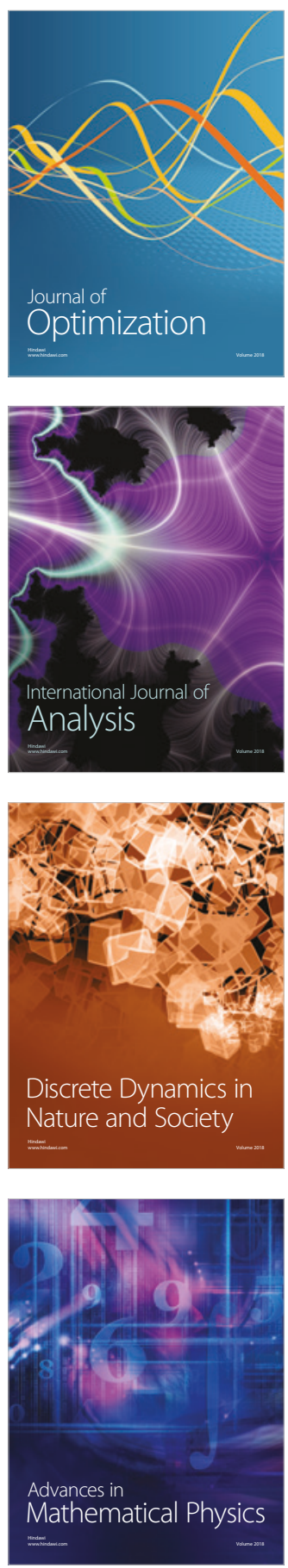